\title{
The Logic of \\ Objective Knowledge and Rational Belief
}

Frans Voorbraak

Department of Pilosophy, University of Utrecht

Logic Group

Preprint Series

No. 55

September 1990
Department of Philosophy University of Utrecht Heidelberglaan 2 3584 CS Utrecht The Netherlands 


\title{
THE LOGIC OF OBJECTIVE KNOWLEDGE AND RATIONAL BELIEF*
}

\author{
Frans Voorbraak \\ Department of Philosophy, University of Utrecht \\ Heidelberglaan 2, 3584 CS Utrecht, The Netherlands
}

\begin{abstract}
In this paper, we study the logical relations between different notions of knowledge and belief by means of a generalization of the usual Kripke models for epistemic logic. We argue that the obtained generalized Kripke models might be useful for carefully distinguishing the many different notions of knowledge and belief. We identify the (highly idealized) notions of objective knowledge and rational (introspective) belief, which correspond with fairly standard notions of knowledge and belief, and propose a system OKRIB for combining both notions which differs essentially from some other such proposals found in the literature. We also consider some other notions of knowledge and belief, and study how they relate to objective knowledge and rational belief.
\end{abstract}

1980 Mathematical Subject Classification: 03A05, 03B45

1987 Computing Review Classification: C.2.4, I.2.3

Key words and phrases: Logics of knowledsge \& belief, distributed systems

September, 1990 .

*The investigations were supported by the Foundation for Philosophical Research (SWON) which is subsidized by the Netherlands Organization for Scientific Research (NWO). 


\title{
THE LOGIC OF OBJECTIVE KNOWLEDGE AND RATIONAL BELIEF
}

\author{
Frans Voorbraak \\ Department of Philosophy, University of Utrecht \\ Heidelberglaan 2, 3584 CS Utrecht, The Netherlands
}

\begin{abstract}
In this paper, we study the logical relations between different notions of knowledge and belief by means of generalizations of the usual Kripke models for epistemic logic. We argue that the obtained generalized Kripke models might be useful for carefully distinguishing the many different notions of knowledge and belief. We identify the (highly idealized) notions of objective knowledge and rational (introspective) belief, which correspond with fairly standard notions of knowledge and belief, and propose a system OKRIB for combining both notions which differs essentially from some other such proposals found in the literature. We also consider some other notions of knowledge and belief, and study how they relate to objective knowledge and rational belief.
\end{abstract}

\section{Introduction}

Recently, there has been a considerable increase in the amount of theory about the logic of knowledge and belief (or epistemic logic), mairly through the impulses of researchers in the field of Artificial Intelligence and Computer Science. One of the main topics of discussion is the problem of logical omniscience, which consists, roughly speaking, of the fact that according to the traditional (possible world) semantics an agent knows/believes the logical consequences (including all tautologies) of anything he knows/believes. This property does not seem to be appropriate for resource-bounded agents, which have only limited reasoning powers.

On account of this problem of logical omniscience various authors have made a distinction between (logically closed) implicit and (not necessarily logically closed) explicit knowledge/belief. In the recent work on epistemic logic much attention is paid to the study of 
different notions of explicit knowledge/belief and their relationship to implicit knowledge/belief. (See Halpern (1986) and Fagin \& Halpern (1988).)

In this paper, the central topic is not the problem of logical omniscience but rather the relation between knowledge and belief. (The problem of logical omniscience will be treated in a sequel of this paper.) Although this relation has already been studied by philosophical logicians and by researchers in AI, we feel there are still a number of unclarified issues here. Since there are a number of different notions of knowledge and belief, an adequate study of the relation between knowledge and belief should first make clear of which notions the relation is studied. Therefore the notions whose interactions are studied in this paper are first treated separately. We concentrate on rather idealized notions of knowledge and belief which we call objective knowledge and rational belief, respectively. However, some other notions of knowledge and belief will also be considered. (All of these notions will be closed under logical consequence, though.)

We essentially use a possible worlds semantics, where the possible worlds are assumed to be complete and consistent. (In the already mentioned sequel of this paper, we allow partial and inconsistent worlds in order to capture beliefs which are not necessarily consistent or logically closed.) Similar approaches have already been discussed by several authors. However, our approach not only differs from these earlier ones in the details of the analysis, but also in the way the semantics is given an intuitive justification. Aspects of the agent's information and belief states are explicitly represented in models intimately related to the traditional Kripke models.

The remainder of this paper is organized as follows: In section 2 we define (generalized) functional Kripke models, sketch the relation between these models and traditional Kripke models, and give some arguments for preferring (generalized) functional Kripke models. The semantics of objective knowledge and rational belief are given in sections 3 and 4, respectively. In section 5 we combine both notions. Section 6 contains a comparison with some other opinions about the interaction of knowledge and belief and a discussion of some alternative notions of knowledge, and in section 7 we discuss alternative (more or less probabilistic) notions of belief. We end with a conclusion.

\section{Functional Kripke models}

Our basic language $\mathcal{L}$ is built up from a set PL of proposition letters (p, q, r,...) and the logical constants $\neg$ and $\wedge$. We will further use the constants $\vee, \supset$ and $\equiv$, which are defined as usual. 
Let for all $i \in\{1,2, \ldots, n\}$ [i] be a modal operator. Then $\mathcal{L}_{[1][2] . .[n]}$ is the smallest extension of $\mathcal{L}$

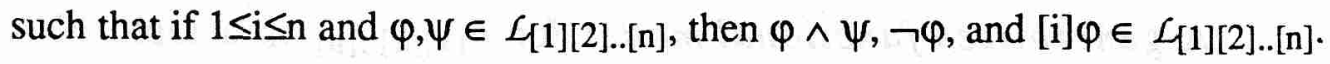

\section{DEFINITION 2.1}

(i) A frame for $\mathcal{L}_{[1][2] . .[n]}$ is a tuple $F=\left\langle W, R_{[1]}, R_{[2]}, \ldots, R_{[n]}\right\rangle$, where $R_{[i]} \subseteq W \times W$.

(ii) A model for $\mathcal{L}_{[1][2] . .[n]}$ is a tuple $M=<w_{0}, W, R_{[1]}, R_{[2]}, \ldots, R_{[n]}, \models>$, where $w_{0} \in W, F=$ $<W, R_{[1]}, R_{[2]}, \ldots, R_{[n]}>$ is a frame for $\mathcal{L}_{[1][2] . .[n]}$ and $\vDash: W \times \mathcal{L}_{[1][2] . .[n]} \rightarrow 2$ such that $\vDash(w,$.$) satisfies the standard propositional clauses and \vDash(w,[i] \varphi)=1 \Leftrightarrow \forall w^{\prime}$ (wR $R_{[i]} w^{\prime}$ $\left.\Rightarrow \vDash\left(w^{\prime}, \varphi\right)=1\right)$. We say that $\mathrm{M}$ is based on $\mathrm{F}$ and $\mathrm{w}_{0}$ is called M's designated world.

(iii) A functional frame for $\mathcal{L}_{[1][2] . .[\mathrm{n}]}$ is a tuple $\mathrm{F}=\left\langle\mathrm{W}, \sigma_{[1]}, \sigma_{[2]}, \ldots, \sigma_{[\mathrm{n}]}\right\rangle$, where $\sigma_{[\mathrm{i}]}: \mathrm{W} \rightarrow$ $\wp \mathrm{W}$.

(iv) A functional model for $\mathcal{L}_{[1][2] . .[\mathrm{n}]}$ is a tuple $\mathrm{M}=\left\langle\mathrm{w}_{0}, \mathrm{~W}, \sigma_{[1]}, \sigma_{[2]}, \ldots, \sigma_{[\mathrm{n}]}, \models>\right.$, where $\mathrm{w}_{0}$ $\in W, F=\left\langle W, \sigma_{[1]}, \sigma_{[2]}, \ldots, \sigma_{[n]}>\right.$ is a functional frame for $\mathcal{L}_{[1][2] . .[n]}$ and $\vDash: W \times \mathcal{L}_{[1][2] . .[n]}$ $\rightarrow 2$ such that $\vDash(w,$.$) satisfies the standard propositional clauses and \vDash(w,[i] \varphi)=1 \Leftrightarrow$ $\forall \mathrm{w}^{\prime} \in \sigma_{[\mathrm{i}]}(\mathrm{w}) \vDash\left(\mathrm{w}^{\prime}, \varphi\right)=1$. We say that $\mathrm{M}$ is based on $\mathrm{F}$ and $\mathrm{w}_{0}$ is called M's designated world.

We often write $w \vDash \varphi$ for $\vDash(w, \varphi)=1$. If $M$ is a (functional) model with valuation function $\vDash$ and designated world $w_{0}$, then $M \models \varphi$ iff $w_{0} \models \varphi$. If $F$ is a (functional) frame, then $F \models \varphi$ iff for every (functional) Kripke model $\mathrm{M}$ based on $\mathrm{F} M \vDash \varphi$. Notice that with every frame we may associate an equivalent functional frame by defining $\sigma_{[i]}(w)=\left\{w^{\prime} \mid w_{[i]} w^{\prime}\right\}$ and with every functional frame we may associate an equivalent frame by defining $w R_{[i]} w^{\prime}$ iff $w^{\prime} \in \sigma_{[i]}(w)$. In fact, we have a 1-1 correspondence between frames/models and functional frames/models. Furthermore, the best known simple properties of the accessibility relations of a traditional Kripke frame correspond with simple properties of the functions in the functional frame associated with the traditional Kripke frame:

PROPOSITION $2.2 \quad$ Let $R \subseteq W \times W$ and let $\sigma: W \rightarrow \wp W$ be given by $\sigma(w)=\left\{w^{\prime} \mid w R w^{\prime}\right\}$.

(i) $\mathrm{R}$ is reflexive iff $\forall \mathrm{w} \in \mathrm{W} w \in \sigma(w)$.

(ii) $\mathrm{R}$ is transitive iff $\forall \mathrm{w}, \mathrm{w}^{\prime} \in \mathrm{W}\left(\mathrm{w} \in \sigma\left(\mathrm{w}^{\prime}\right) \Rightarrow \sigma(\mathrm{w}) \subseteq \sigma\left(\mathrm{w}^{\prime}\right)\right)$.

(iii) $\mathrm{R}$ is euclidean iff $\forall \mathrm{w}, \mathrm{w}^{\prime}, \mathrm{w}^{\prime \prime} \in \mathrm{W}$ ( $\mathrm{w}^{\prime}, \mathrm{w}^{\prime \prime} \in \sigma(\mathrm{w}) \Rightarrow \mathrm{w}^{\prime \prime} \in \sigma\left(\mathrm{w}^{\prime}\right)$ )).

(iv) $\mathrm{R}$ is transitive and euclidean iff $\forall \mathrm{w}^{\prime} \mathrm{w}^{\prime} \in \mathrm{W}\left(\mathrm{w} \in \sigma\left(\mathrm{w}^{\prime}\right) \Rightarrow \sigma(\mathrm{w})=\sigma\left(\mathrm{w}^{\prime}\right)\right)$.

Proof. Trivial.

The reason for introducing functional Kripke models is that they form a bridge between the traditional Kripke models and the general kind of models we propose for epistemic logic: 
DEFINITION 2.3 A generalized functional model for $\mathcal{L}_{[1][2] . .[n]}$ is a tuple $\mathbf{M}=$ $<\mathrm{w}_{0}, \mathrm{~W}, \Sigma_{[1]}, \Sigma_{[2]}, \ldots, \Sigma_{[\mathrm{n}]}, \sigma_{[1]}, \sigma_{[2]}, \ldots, \sigma_{[\mathrm{n}]}, \mathrm{F}_{[1]}, \mathrm{F}_{[2]}, \ldots, \mathrm{F}_{[\mathrm{n}]}, \vDash>$, where $\mathrm{w}_{0} \in \mathrm{W}, \Sigma_{[\mathrm{i}]}$ is a nonempty set, $\sigma_{[\mathrm{i}]}: \mathrm{W} \rightarrow \Sigma_{[\mathrm{i}]}, \mathrm{F}_{[\mathrm{i}]}$ is a set of functions with domain $\Sigma_{[\mathrm{i}]}$, and $\vDash: \mathrm{W} \times \mathcal{L}_{[1][2] . .[\mathrm{n}]} \rightarrow$ 2 such that $\vDash(w,$.$) satisfies the standard propositional clauses and \vDash(w,[i] \varphi)$ functionally depends on $\vDash i W \times[\varphi], \sigma_{[i]}, w$, and (a subset $F$ of) $F_{[i]}$.

$\Sigma_{[i]}$ denotes a set of epistemic states, $\sigma_{[i]}$ associates with every world an element of $\Sigma_{[i]}$, and for every epistemic state $S \in \Sigma_{[i]}$ and $f \in F_{[i]} f(S)$ denotes an aspect of the epistemic state $S$. Elements of $\mathrm{F}_{[\mathrm{i}]}$ are called information extracting functions or projection functions, since they extract information out of an epistemic state by "projecting" the epistemic state onto some aspect of the state. An example of a projection function is the function $\|\|:. \Sigma_{[i]} \rightarrow \wp \mathrm{W}$ given by $\|S\|=$ $\{\mathrm{w} \in \mathrm{W} I \mathrm{w}$ is possible according to $\mathrm{S}\}$. Another example is the function $\Pi$ such that $\Pi(\mathrm{S})$ yields a probability measure on $\wp\|S\|$ giving the probabilities which according to $S$ have to be assigned to the (sets of) possible worlds. In general, we will only exhibit those projection functions which are mentioned in the valuation clauses of the modalities or which are used to single out a particular class of generalized functional models.

Generalized functional models generalize functional models in two respects: (1) epistemic states occur as atomic entities in the generalized models; they are no longer (indirectly) represented by means of sets of possible worlds and (2) in the generalized models modal operators may have nonstandard valuation clauses. Nevertheless, a class of generalized functional models will often turn out to be equivalent to a class of functional models which correspond with traditional Kripke models, so one can often invoke well-known results and methods to obtain soundness and completeness results.

In the following section, an example of the use of non-standard valuation clauses will be given. Our justification for the explicit representation of epistemic states in the generalized functional models is that it helps to systematize the study of the relations between various epistemic modalities. For example, the two central notions in this paper - objective knowledge and rational belief - refer to different epistemic states, and are therefore not as strongly related as some authors seem to think, whereas some (strongly related) notions of knowledge and belief might be considered to refer to different aspects of the same epistemic state. (Cf. sections 5 and 6.) In general, we believe the generalized functional models to be useful in bringing the study of epistemic logic to a higher level of 'informal rigour' (in the sense of Kreisel). 


\section{Objective knowledge}

Let $\mathrm{K}_{\alpha}$ be the operator with the following intuitive meaning:

$\mathrm{K}_{\alpha} \varphi$ : agent $\alpha$ objectively knows that $\varphi$, i.e. $\varphi$ is the case (in every world that is possible) given the information available to $\alpha$.

Since we will restrict ourselves to the one agent case, we will usually omit the subscript. The notion of objective knowledge applies to any agent which is capable of processing information; we do not require the agent to consider the (im)possibility of some worlds. The logic of this notion of objective knowledge will be denoted by $\mathrm{OK}$. Closely following the above intuitive explanation of the meaning of the operator $\mathrm{K}$, we propose the following semantics for $\mathrm{K}$ :

DEFINITION 3.1 An information state model is a generalized functional model $\mathbf{M}=$ $<\mathrm{w}_{0}, \mathrm{~W}, \Sigma_{\mathrm{K}}, \kappa, \models>$, where $\mathrm{w} \models \mathrm{K} \varphi \Leftrightarrow \forall \mathrm{w}^{\prime} \in \mathrm{W}\left(\kappa\left(\mathrm{w}^{\prime}\right)=\kappa(\mathrm{w}) \Rightarrow \mathrm{w}^{\prime} \models \varphi\right)$. Elements of $\Sigma_{\mathrm{K}}$ are called information states.

The class of information state models is easily seen to be equivalent to a class of functional models which we call OK-models and which correspond with Kripke models for S5.

\section{DEFINITION 3.2}

(i) An $\boldsymbol{O K}$-frame is a functional frame $\mathrm{F}=\langle\mathrm{W}, \mathrm{K}\rangle$ for $\mathcal{L}_{\mathrm{K}}$ such that

1. $\forall \mathrm{w} \in \mathrm{W}$ w $\in \kappa(\mathrm{w})$

2. $\forall \mathrm{w}, \mathrm{w}^{\prime} \in \mathrm{W}\left(\mathrm{w} \in \kappa\left(\mathrm{w}^{\prime}\right) \Rightarrow \kappa(\mathrm{w})=\kappa\left(\mathrm{w}^{\prime}\right)\right)$

(ii) An $\boldsymbol{O K}$-model is a functional model based on an $\mathrm{OK}$-frame.

PROPOSITION 3.3 Let $\varphi \in \mathcal{L}_{\mathrm{K}}$. The following are equivalent:

(i) For every information state módel $M \quad M \vDash \varphi$.

(ii) $\vDash \mathrm{OK} \varphi$.

(iii) $\vDash s 5 \varphi$.

Proof. Let $\mathrm{M}=\left\langle\mathrm{w}_{0}, \mathrm{~W}, \Sigma_{\mathrm{k}}, \mathrm{K}, \models>\right.$ be an information state model. Define $\mathrm{R} \subseteq \mathrm{W} \times \mathrm{W}$ by $\mathrm{wRw}^{\prime}$ iff $\kappa(w)=\kappa\left(w^{\prime}\right)$. Then $M^{\prime}=\left\langle w_{0}, W, R, \models>\right.$ is an S5 Kripke model equivalent to $M$. On the other hand, with every $S 5$ Kripke model $M=\left\langle w_{0}, W, R, \models>\right.$ one may associate an equivalent OK-model $M^{\prime}=\left\langle w_{0}, W, \kappa, \models>\right.$ by $\kappa(w)=\left\{w^{\prime} \in W \mid w_{R} w^{\prime}\right\}$. Finally, if $M=\left\langle w_{0}, W, \kappa, \models>\right.$ is an $\mathrm{OK}$-model, then $\mathrm{M}^{\prime}=\left\langle\mathrm{w}_{0}, \mathrm{~W}, \wp \mathrm{W}, \mathrm{K}, \models>\right.$ is an equivalent information state model. 
Notice that the notion of information state does not have to be specified in order to determine the logic of $\mathrm{K}$. The equivalence of information state models and $\mathrm{OK}$-models of course does not show that information states can be equated with sets of possible worlds, since the equivalence depends for example on the fact that that the language $\mathcal{L}_{\mathrm{K}}$ does not admit one to exploit probabilistic information which may be contained in the information states.

The above method of identifying the logic of objective knowledge as S5 is a generalization of the method by which the logic of the knowledge of processors in a distributed system is identified to be S5. In the latter case, one takes the information state of a processor to be identical to the internal state of the processor. Notice that although the introspection axioms $(\mathrm{K} \varphi \supset \mathrm{KK} \varphi$ and $\neg \mathrm{K} \varphi \supset \mathrm{K} \neg \mathrm{K} \varphi$ ) are valid in $\mathrm{OK}$, we did not assume our agents to have some kind of privileged access to or a perfect awareness of their epistemic attitudes.

\section{Rational (introspective) belief}

In this section we consider the operator $\mathrm{B}_{\alpha}$ with the following intuitive meaning:

$B_{\alpha} \varphi$ : agent $\alpha$ rationally believes (is rationally convinced) that $\varphi$, i.e. $\varphi$ is valid in every world that is considered possible by $\alpha$.

Again we usually omit the subscript. Notice that the above notion of belief is a very strong one. In fact, if $\mathrm{B}_{\alpha} \varphi$, then $\alpha$ will be inclined to express his belief by saying that he knows that $\varphi$. If an agent says that he (only) believes that $\varphi$, then most likely a weaker notion of belief is used. Some possible weaker notions are treated in section 7 .

Let $\beta(w)$ be the rational belief state (of an agent $\alpha$ ) in w and remember that for any epistemic state $S$ the set of worlds which are possible according to $S$ is denoted by $\|S\|$. Hence $\|\beta(w)\|$ denotes the set of worlds which are considered possible (by $\alpha$ ) in w. Rational belief states are assumed to satisfy the following two conditions:

CONSISTENCY: For every rational belief state $S \quad\|S\| \neq \varnothing$. INTROSPECTION: For every rational belief state $S(w \in\|S\| \Rightarrow \beta(w)=S)$.

The consistency-condition derives from the assumed rationality of the agent: a rational agent will not adhere to an inconsistent șet of beliefs, but will revise this set to a consistent one. The introspection-condition derives from the assumption that a rational agent is fully aware of his own belief state. Hence in every world he considers to be a possible candidate of the actual world he must have the same belief state. A rational agent is not infallible, hence we do not 
require $w \in\|\beta(w)\|$. Some other conditions on rational belief states (concerning the relation between rational belief states and information states) are treated in the following section.

Notice that we do not equate a rational agent's belief state $S$ with IISI, since we do not want to exclude the possibility that the agent has e.g. beliefs concerning the relative likelihood of subclasses of ISII. RIB, the logic of rational introspective belief has the following semantics:

DEFINITION 4.1 An belief state model is a generalized functional model $\mathrm{M}=$ $<\mathrm{w}_{0}, \mathrm{~W}, \Sigma_{\mathrm{B}}, \beta, \vDash>$, which satisfies

$$
\begin{array}{ll}
\text { 1. } & \forall w \in W\|\beta(w)\| \neq \varnothing \\
\text { 2. } & \forall w, w^{\prime} \in \mathrm{W}\left(w \in\left\|\beta\left(w^{\prime}\right)\right\| \Rightarrow \beta(w)=\beta\left(w^{\prime}\right)\right) \\
\text { 3. } & w \models B \varphi \Leftrightarrow \forall w^{\prime} \in\|\beta(w)\| w^{\prime} \models \varphi .
\end{array}
$$

Elements of $\Sigma_{\mathrm{B}}$ are called belief states.

Just as in the case of the information state models, the class of belief state models is equivalent to a class of functional models which correspond with very familiar Kripke models. These functional models are called RIB-models and they correspond with Kripke models for KD45.

\section{DEFINITION 4.2}

(i) A RIB-frame is a functional frame $\mathrm{F}=\left\langle\mathrm{W}, \beta>\right.$ for $\mathcal{L}_{\mathrm{B}}$ such that

1. $\forall \mathrm{w} \in \mathrm{W} \beta(\mathrm{w}) \neq \varnothing$

2. $\forall w, w^{\prime} \in W\left(w \in \beta\left(w^{\prime}\right) \Rightarrow \beta(w)=\beta\left(w^{\prime}\right)\right)$.

(ii) A RIB-model is a functional model based on a RIB-frame.

PROPOSITION 4.3 Let $\varphi \in \mathcal{L}_{\mathrm{B}}$. The following are equivalent:

(i) For every belief state model $\mathrm{M} \quad \mathrm{M} \models \varphi$.

(ii) $\vDash \mathbf{R I B} \varphi$.

(iii) $\models_{\mathrm{KD} 45} \varphi$.

Proof. Similar to 3.3 .

\section{Combining knowledge and belief}

The information objectively available to a rational introspective agent surely contains all information about his own rational belief state. Hence if a world $w^{\prime}$ is possible given the information available to an agent $\alpha$ in world $w$, then $\alpha$ 's rational belief state in $w$ ' is identical to that in w. Further, if a world $w$ is a member of $\|S\|$, for some rational belief state of agent $\alpha$, 
then it is reasonable to assume that the set of worlds which are possible according to $\alpha$ 's information state in $w$ is a subset of $\|S\|$. (A world which is considered(!) to be objectively possible from a world believed to be a possible candidate for the actual world should itself be believed to be a possible candidate for the actual world.) Hence we arrive at the following additional assumptions on the relation between information classes and rational belief classes:

OBJECTIVITY: $w \in\left\|\kappa\left(w^{\prime}\right)\right\| \Rightarrow \beta(w)=\beta\left(w^{\prime}\right)$.

BELIEVED SOUNDNESS: $w \in\left\|\beta\left(w^{\prime}\right)\right\| \Rightarrow\|\kappa(w)\| \subseteq\left\|\beta\left(w^{\prime}\right)\right\|$.

Another interesting assumption is the following:

BELIEVED COMPLETENESS: $w \in\left\|\beta\left(w^{\prime}\right)\right\| \Rightarrow\|\kappa(w)\| \supseteq\left\|\beta\left(w^{\prime}\right)\right\|$.

This assumption might be appropriate for an agent who is very confident in the sense that he believes to have fully exploited the information available to him. However, in general we will not assume believed completeness. The semantics for $\mathbf{O K} \&(\mathbf{O}) \mathbf{R I B}$, the system in which objective knowledge and (overconfident) rational introspective belief are combined, becomes:

DEFINITION 5.1 An information \& belief state model is a generalized functional model $\mathrm{M}$ $=\left\langle w_{0}, W, \Sigma_{K}, \Sigma_{B}, \kappa, \beta,\|.\|_{K},\|.\|_{B}, \vDash>\right.$ such that $\left\langle w_{0}, W, \Sigma_{K}, \kappa, \vDash \uparrow \mathcal{L}_{K}\right\rangle$ is a information state model, $<\mathrm{w}_{0}, \mathrm{~W}, \Sigma_{\mathrm{B}}, \beta, \vDash\left\lceil\mathcal{L}_{\mathrm{B}}>\right.$ is a belief state model, $\|.\|_{\mathrm{K}}: \Sigma_{\mathrm{K}} \rightarrow \wp \mathrm{W},\|.\|_{\mathrm{B}}: \Sigma_{\mathrm{B}} \rightarrow \wp \mathrm{W}$, and for all $w, w^{\prime} \in W: w \in\left\|\kappa\left(w^{\prime}\right)\right\| \Rightarrow \beta(w)=\beta\left(w^{\prime}\right)$ and $w \in\left\|\beta\left(w^{\prime}\right)\right\| \Rightarrow\|\kappa(w)\| \subseteq\left\|\beta\left(w^{\prime}\right)\right\|$. An information \& overconfident belief state model is an information \& belief model which additionally satisfies $w \in\left\|\beta\left(w^{\prime}\right)\right\| \Rightarrow\|\kappa(w)\| \supseteq\left\|\beta\left(w^{\prime}\right)\right\|$.

DEFINITION 5.2

(i) An $O K \& R I B$-frame is a tuple $F=\langle\mathrm{W}, \kappa, \beta\rangle$, where

1. $\langle\mathrm{W}, \mathrm{K}\rangle$ is an $\mathrm{OK}$-frame

2. $<W, \beta>$ is a RIB-frame

3. $\forall \mathrm{w}, \mathrm{w}^{\prime} \in \mathrm{W}\left(\mathrm{w} \in \mathrm{k}\left(\mathrm{w}^{\prime}\right) \Rightarrow \beta(\mathrm{w})=\beta\left(\mathrm{w}^{\prime}\right)\right)$

4. $\forall \mathrm{w}, \mathrm{w}^{\prime} \in \mathrm{W}\left(\mathrm{w} \in \beta\left(\mathrm{w}^{\prime}\right) \Rightarrow \mathrm{k}(\mathrm{w}) \subseteq \beta\left(\mathrm{w}^{\prime}\right)\right)$.

(ii) An $O K \& R I B$-model is a functional model based on an OK\&RIB-frame.

(iii) An $O K \& O R I B$-frame (model) is an OK\&RIB-frame (model) which additionally satisfies $\forall w, w^{\prime} \in W\left(w \in \beta\left(w^{\prime}\right) \Rightarrow \kappa(w) \supseteq \beta\left(w^{\prime}\right)\right)$.

OK\& $(\mathbf{O}) \mathbf{R I B}$-models are equivalent to information \& (overconfident) belief state models and just as in the case of the OK- and the RIB-models, the OK\& $(O) R I B$-models correspond to a relatively easy characterizable class of Kripke models: 
PROPOSITION 5.3 Let $\varphi \in \mathcal{L}_{\mathrm{KB}}$. The following are equivalent:

(i) For every information \& belief state model $M \quad M=\varphi$.

(ii) $\vDash$ OK\&RIB $\varphi$.

(iii) $\varphi$ is valid in every model for $\mathcal{L}_{K B}$ such that $R_{K}$ is an equivalence relation, $R_{B}$ is serial, transitive, and euclidean, and $R_{B} R_{K} \subseteq R_{B} \supseteq R_{K} R_{B}$. (Since $R_{K}$ is reflexive, the inclusions may be replaced by identities.)

PROPOSITION 5.4 Let $\varphi \in \mathcal{L}_{\mathrm{KB}}$. The following are equivalent:

(i) For every information \& overconfident belief state model $M \quad M \vDash \varphi$.

(ii) $\vDash$ OK\&ORIB $\varphi$.

(iii) $\varphi$ is valid in every model for $\mathcal{L}_{\mathrm{KB}}$ such that $R_{K}$ is an equivalence relation, $R_{B}$ is serial, transitive, and euclidean, $R_{B} R_{K} \subseteq R_{B} \supseteq R_{K} R_{B}$, and $\forall x y z\left(x R_{B} y \& x R_{B} z \Rightarrow y R_{K} z\right)$.

(The proofs of 5.3 and 5.4 are similar to that of 3.3)

\section{LEMMA 5.5}

(i) $\mathrm{B} \varphi \supset \mathrm{BK} \varphi$ corresponds to $\mathrm{R}_{\mathrm{B}} \mathrm{R}_{\mathrm{K}} \subseteq \mathrm{R}_{\mathrm{B}}$.

(ii) $\mathrm{B} \varphi \supset \mathrm{KB} \varphi$ corresponds to $R_{K} R_{B} \subseteq R_{B}$.

(iii) $\neg \mathrm{B} \varphi \supset \mathrm{B} \neg \mathrm{K} \varphi$ corresponds to $\forall \mathrm{xyz}\left(\mathrm{x} R_{\mathrm{B}} \mathrm{y} \& \mathrm{x} \mathrm{R}_{\mathrm{B}} \mathrm{y} \Rightarrow \mathrm{y} \mathrm{R}_{\mathrm{K}} \mathrm{z}\right.$ ).

Proof. (i) and (ii) are well-known. (iii) is proved in the standard way:

Consider a model $\mathrm{M}$ for $\mathcal{L}_{\mathrm{KB}}$ which satisfies $\forall \mathrm{xyz}\left(\mathrm{x} R_{\mathrm{B}} \mathrm{y} \& \mathrm{x} \mathrm{R}_{\mathrm{B}} \mathrm{z} \Rightarrow \mathrm{x} \mathrm{R}_{\mathrm{K}} \mathrm{z}\right.$ ) and assume w0 $\vDash \neg B \varphi$. Then there exists a $w$ such that $w_{0} R_{B} w$ and $w \vDash \neg \varphi$. Since $w_{0} R_{B} w '$ implies $w ' R_{K} w$, we have $w_{0}=\mathrm{B} \neg \mathrm{K} \varphi$.

On the other hand, consider a frame $\mathrm{F}=\left\langle\mathrm{W}, \mathrm{R}_{\mathrm{K}}, \mathrm{R}_{\mathrm{B}}>\right.$ for $\mathcal{L}_{\mathrm{KB}}$ which does not satisfy $\forall \mathrm{xyz}$ $\left(x R_{B} y \& x R_{B} z \Rightarrow y R_{K} z\right)$. Then for some $w, w^{\prime}, w " \in W$ we have $w R_{B} w^{\prime}, w R_{B} w^{\prime \prime}$ and $\neg \mathrm{w} \mathrm{R}_{\mathrm{K}} \mathrm{w}$. Let $\mathrm{M}$ be a functional model based on $\mathrm{F}$ such that $\mathrm{w}$ is M's designated world and $\left.\vDash(x, p)=1 \Leftrightarrow x \in W \backslash w^{\prime \prime}\right\}$. Then $M \not \neg \neg B p \supset B \neg K p$.

\section{DEFINITION 5.6}

(i) OK\&RIB is the system in $\mathcal{L}_{\mathrm{KB}}$ given by the following rules and axioms:

Modus Ponens, Necessitation for $\mathrm{K}$ and $\mathrm{B}$, all propositional tautologies in $\mathcal{L}_{\mathrm{KB}}$, Distribution for K and B, 4 ( $\square \varphi \supset \square \square \varphi)$ for K and B, $5(\neg \square \varphi \supset \square \neg \square \varphi)$ for K and B, $\mathrm{K} \varphi \supset \varphi, \mathrm{B} \varphi \supset \neg \mathrm{B} \neg \varphi, \mathrm{B} \varphi \supset \mathrm{BK} \varphi, \mathrm{B} \varphi \supset \mathrm{KB} \varphi$.

(ii) $\quad \mathrm{OK} \& O R I B=O K \& R I B+\neg B \varphi \supset B \neg K \varphi$.

PROPOSITION 5.7 (i) $\quad \forall \varphi \in \mathcal{L}_{\mathrm{KB}}(\vdash \mathrm{OK} \& \operatorname{RIB} \varphi \Leftrightarrow \vDash$ OK\&RIB $\varphi)$

(ii) $\forall \varphi \in \mathcal{L}_{\mathrm{KB}}(\vdash \operatorname{OK} \& \operatorname{ORIB} \varphi \Leftrightarrow \models \operatorname{OK} \& \operatorname{ORIB} \varphi)$ 
Proof. Immediate from Lemma 5.5 and other well-known results.

LEMMA 5.8 The following are theorems of OK\&RIB:

(i) $\mathrm{KK} \varphi \equiv \mathrm{K} \varphi$

(v) $\mathrm{KB} \varphi \equiv \mathrm{B} \varphi$

(ii) $\mathrm{K} \neg \mathrm{K} \varphi \equiv \neg \mathrm{K} \varphi$

(vi) $\mathrm{K} \neg \mathrm{B} \varphi \equiv \neg \mathrm{B} \varphi$

(iii) $\mathrm{BB} \varphi \equiv \mathrm{B} \varphi$

(vii) $\mathrm{BK} \varphi \equiv \mathrm{B} \varphi$

(iv) $\mathrm{B} \neg \mathrm{B} \varphi \equiv \neg \mathrm{B} \varphi$

(viii) $(\mathrm{B} \neg \varphi \supset \mathrm{B} \neg \mathrm{K} \varphi) \wedge(\mathrm{B} \neg \mathrm{K} \varphi \supset \neg \mathrm{B} \varphi)$

Proof. Only two cases are not completely trivial:

(vi) $\neg \mathrm{B} \varphi \supset \mathrm{K} \neg \mathrm{B} \varphi$ : follows from (v) and the S5-property $\psi \supset \mathrm{K} \neg \mathrm{K} \neg \psi$ applied to $\psi=\neg \mathrm{B} \varphi$.

(viii) $(\mathrm{B} \neg \varphi \supset \mathrm{B} \neg \mathrm{K} \varphi$ ) follows from (vii) and $\mathrm{K} \varphi \supset \neg \mathrm{K} \neg \varphi$;

$(\mathrm{B} \neg \mathrm{K} \varphi \supset \neg \mathrm{B} \varphi$ ) follows from (vii) and $\mathrm{B} \varphi \supset \neg \mathrm{B} \neg \varphi$.

\section{PROPOSITION 5.8}

(i) OK\&RIB has 12 distinct modalities, viz. $\cdot, \mathrm{K}, \mathrm{B}, \mathrm{K} \neg, \mathrm{B} \neg, \mathrm{B} \neg \mathrm{K}$, and their negations. The implications among them are pictured in figure 1 below.

(ii) OK\&ORIB has 10 distinct modalities, viz. $\cdot \mathrm{K}, \mathrm{B}, \mathrm{K} \neg, \mathrm{B} \neg$, and their negations. The implications among them are pictured in figure 2 below.

Proof. Immediate from lemma 5.7.

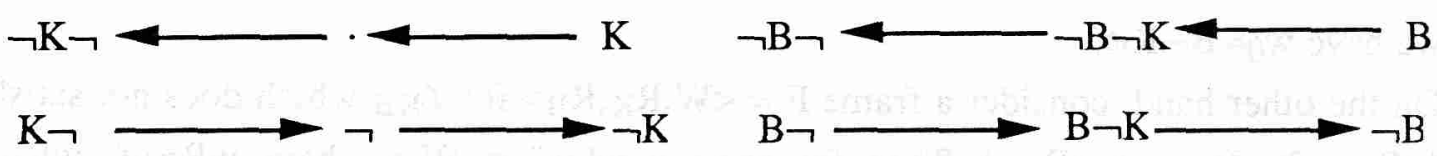

FIGURE 1. Modalities of OK\&RIB.

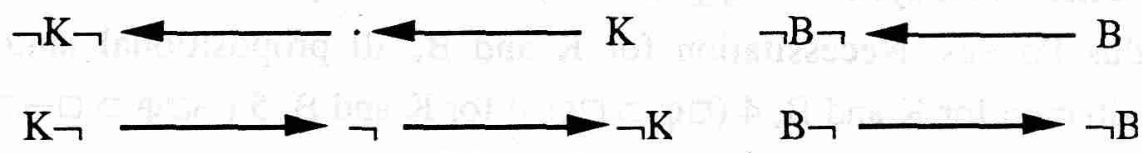

FIGURE 2. Modalities of OK\&ORIB. 


\section{Knowledge which implies rational belief}

It is no surprise that $\mathbf{O K \& R I B}$ is not consistent with the ideas about the relation between knowledge and belief in Hintikka (1962) the notions of objective knowledge and rational belief are rather different from the originally studied epistemic notions. However, objective knowledge and rational belief do seem to correspond with the nowadays predominant notions of knowledge and belief. Nevertheless, OK\&RIB differs in a number of respects from the system which was proposed in Kraus \& Lehmann (1986) and thoroughly studied from a technical point of view in van der Hoek (1989). This system - let us call it KL - is more extensive than OK\&RIB, since it also treats the notions of common knowledge and common belief. As far as pure knowledge propositions (i.e. propositions containing no other modal operators than $\mathrm{K}$ ) and pure belief propositions are concerned, KL and $\mathrm{OK} \& \mathrm{RIB}$ agree. However, the $\mathbf{O K \& R I B}$ theorem $\mathrm{B} \varphi \supset \mathrm{BK} \varphi$ is not derivable in $\mathbf{K L}$, and adding it to $\mathbf{K L}$ would lead to the derivability of $B \varphi \supset K \varphi$.

As already observed by Wolfgang Lenzen (1978, p.80), $\mathrm{B} \varphi \supset \mathrm{BK} \varphi$ is only reasonable in case $\mathrm{B}_{\alpha} \varphi$ stands for " $\alpha$ is convinced that $\varphi$ " and not in case $\mathrm{B}_{\alpha} \varphi$ means something like " $\alpha$ presumes that $\varphi$ ". Hence at first sight the non-derivability of $\mathrm{B} \varphi \supset \mathrm{BK} \varphi$ in KL might be defended by arguing that KL does not intend to capture the notion of conviction. However, since an agent may be cautious and only presume something if he is convinced of it, the fact that $\mathrm{KL}+(\mathrm{B} \supset \mathrm{BK}) \vdash \mathrm{B} \varphi \supset \mathrm{K} \varphi$ indicates that there is something wrong with $K L$. Another indication is provided by the fact that $B K \varphi \supset K \varphi$ is a theorem of $K L$. This principle contradicts the intuition that you might believe you know that $\varphi$, while in fact $\varphi$ is not true. In a sense, the principle is self-defeating: Let $\psi$ be the sentence "BK $\varphi \supset \mathrm{K} \varphi$ is not valid". The author of this paper believes to know that $\psi$. Hence $B K \psi$, where the author is the agent of $\mathrm{K}$ and $\mathrm{B}$. But then the validity of $\mathrm{BK} \varphi \supset \mathrm{K} \varphi$ implies its invalidity.

KL has $\mathrm{K} \varphi \supset \mathrm{B} \varphi$ among its axioms. Deleting this axiom (which is not valid in OK\&RIB) would block both the derivation of $B K \varphi \supset K \varphi$ in $K L$ and the derivation of $B \varphi \supset$ $\mathrm{K} \varphi$ in $\mathbf{K L}+(\mathrm{B} \supset \mathrm{BK})$. However, one frequently seems to use a notion of knowledge which does imply belief. For example, in the philosophical literature knowledge is often equated with (justified) true belief. Such a notion of knowledge $\mathrm{K}^{+}$is obviously different from our notion of objective knowledge and the justification given in section 3 for taking $\mathbf{S 5}$ as the logic of $\mathrm{K}$ does not apply to $\mathrm{K}^{+}$. In fact, if one assumes $\mathrm{K}^{+} \varphi \supset \mathrm{B} \varphi$, then the negative introspection principle for $\mathrm{K}^{+}\left(\neg \mathrm{K}^{+} \varphi \supset \mathrm{K}^{+} \neg \mathrm{K}^{+} \varphi\right)$ no longer seems acceptable, since it would make it possible to derive $\mathrm{BK}^{+} \varphi \supset \mathrm{K}^{+} \varphi$ from the principle $\mathrm{B} \neg \psi \supset \neg \mathrm{B} \psi$.

Similar arguments are given in Lenzen (1978), where it is argued that the logic of justified true belief is at least as strong as $\mathbf{S 4 . 2}(=\mathbf{S 4}+\neg \square \neg \square \varphi \supset \square \neg \square \neg \varphi)$ and at most as strong as S4.4 (= S4 $+\varphi \supset(\neg \square \neg \square \varphi \supset \square \varphi))$. The latter system is claimed to be the logic of 
true belief. Indeed it is not difficult to check that if we define in $\mathcal{L}_{\mathrm{B}} \mathrm{K}^{*} \varphi=\mathrm{B} \varphi \wedge \varphi$, then $\forall \varphi \in$ $\mathcal{L}_{\mathrm{K}^{*}}\left(\vDash \mathbf{R I B} \varphi\right.$ iff $\left.\vDash_{\mathbf{S} 4.4} \varphi\right)$.

In $\mathcal{L}_{\mathrm{KB}}$ we can define another notion of knowledge which implies belief: $\mathrm{K}^{\prime} \varphi=\mathrm{K} \varphi \wedge$ $\mathrm{B} \varphi$. Below we show that the logic of this notion of rationally believed objective knowledge (ROK) lies between S4.3 and S4.4:

DEFINITION 6.1 A $R O K$-model is a Kripke model for $\mathcal{L}_{K^{\prime}}$ s.t. $R_{K^{\prime}}=R_{1} \cup R_{2}, R_{1}$ is an equivalence relation, $R_{2}$ is serial, transitive and euclidean and $R_{1} R_{2}=R_{2}=R_{2} R_{1}$.

DEFINITION 6.2 A relation $R$ is called semi-euclidean iff $\forall x y z((x R y \wedge x R z) \rightarrow(y R z \vee$ $\mathrm{zRx})$ ).

\section{LEMMA 6.3}

(i) If $R=R_{1} \cup R_{2}$, where $R_{1}$ is an equivalence relation and $R_{2}$ is serial, transitive, euclidean, and $R_{1} R_{2}=R_{2}=R_{2} R_{1}$, then $R$ is reflexive, transitive, and semi-euclidean.

(ii) If $R$ is reflexive, transitive, and semi-euclidean, then there exists $R_{1}$ and $R_{2}$ such that $R$ $=R_{1} \cup R_{2}, R_{1}$ is an equivalence relation and $R_{2}$ is serial, transitive and euclidean, and $R_{1} R_{2}=$ $\mathrm{R}_{2}=\mathrm{R}_{2} \mathrm{R}_{1}$.

Proof. (i) Trivial. (ii) Define $\mathrm{xR}_{1} \mathrm{y}$ iff $\mathrm{x}=\mathrm{y} \vee(\mathrm{xRy} \wedge \exists \mathrm{z}(\mathrm{xRz} \wedge \neg \mathrm{zRy})) \& \mathrm{xR}_{2} \mathrm{y}$ iff $\forall \mathrm{z}(\mathrm{xRz} \rightarrow$ $z R y$ ). Then $R_{1}$ and $R_{2}$ satisfy the requirements of (ii).

LEMMA 6.4 The transitive, semi-euclidean Kripke frames for $\mathcal{L}_{\square}$ are characterized by the set $S=\left\{\square \varphi \supset \square \square \varphi,(\varphi \wedge \neg \square \varphi \wedge \square(\varphi \vee \square(\varphi \supset \square \varphi))) \supset \square \neg \square \varphi \mid \varphi \in \mathcal{L}_{\square}\right\}$.

Proof. It is easily checked that every formula of $\mathrm{S}$ is valid in every transitive, semi-euclidean frame. On the other hand, let $F=\left\langle\mathrm{W}, \mathrm{R}>\right.$ be a transitive frame for $\mathcal{L}_{\square}$. (It is well-known that there is a countermodel against $\square p \supset \square \square$ p based on any non-transitive frame.) Assume that $R$ is not semi-euclidean. Then there exist $w, w^{\prime}, w^{\prime \prime} \in W_{\text {such }}$ that $w^{\prime} w^{\prime}, w R w^{\prime \prime}, \neg w^{\prime} R w^{\prime \prime}$, and $\neg \mathrm{w} " \mathrm{Rw}$. Let $\mathrm{M}=<\mathrm{w}, \mathrm{W}, \mathrm{R},>$ be a functional model such that $\models(\mathrm{x}, \mathrm{p})=1$ iff $\mathrm{x}=\mathrm{w}$ or $\mathrm{xRw}$ or $\neg \mathrm{xRw}$ " or $\mathrm{x} \neq \mathrm{w}$ ". Then $\mathrm{M} \not(\mathrm{p} \wedge \neg \square \mathrm{p} \wedge \square(\mathrm{p} \vee \square(\mathrm{p} \supset \square \mathrm{p}))) \supset \square \neg \square \mathrm{p}$.

PROPOSITION 6.5 Let $\varphi \in \mathcal{L}_{\mathrm{K}}$. The following are equivalent:

(i) $\quad \models \mathbf{R O K} \varphi$.

(ii) $\quad \vDash \mathbf{K T 4 5}^{\prime} \varphi$, where $\left(5^{\prime}\right)=\left(\varphi \wedge \neg K^{\prime} \varphi \wedge K^{\prime}\left(\varphi \vee K^{\prime}\left(\varphi \supset K^{\prime} \varphi\right)\right)\right) \supset K^{\prime} \neg K^{\prime} \varphi$.

(iii) For every reflexive, transitive, semi-euclidean Kripke model $M$ for $\mathcal{L}_{K^{\prime}} M \models \varphi$.

Proof. Immediate from 6.3 and 6.4 . 
PROPOSITION 6.6 KT45' is (i) a proper extension of $\mathbf{S 4 . 3}(=\mathbf{S} 4+\square(\square \varphi \supset \square \psi) \vee \square(\square \psi$ $\supset \square \varphi)$ ) and (ii) a proper subsystem of $\mathbf{S 4 . 4}$.

Proof. (i) If $\mathrm{R}$ is semi-euclidean and transitive, then $\mathrm{R}$ is connected, i.e. ( $\mathrm{xRy} \& \mathrm{xRz}) \Rightarrow(\mathrm{yRz}$ or $\mathrm{zRy}$ ). $\mathrm{S4.3}$ is sound and complete with respect to reflexive transitive connected Kripke models. Hence KT45' is an extension of S4.3. The following S4.3 countermodel against (5') shows that the inclusion is proper: $M=\left\langle\mathrm{w}_{0}, \mathrm{~W}, \mathrm{R}, \mathrm{I}\right\rangle$, with $\mathrm{W}=\left\{\mathrm{w}_{0}, \mathrm{w}_{1}, \mathrm{w}_{2}\right\}, \mathrm{R}=$ $\mathrm{W} \times \mathrm{W} \backslash\left\{\left(\mathrm{w}_{1}, \mathrm{w}_{0}\right),\left(\mathrm{w}_{2}, \mathrm{w}_{0}\right),\left(\mathrm{w}_{2}, \mathrm{w}_{1}\right)\right\}, \mathrm{I}: \mathrm{W} \times \mathrm{PL} \rightarrow 2$ is given by $\mathrm{I}\left(\left(\mathrm{w}_{0}, \mathrm{p}\right)=\mathrm{I}\left(\mathrm{w}_{2}, \mathrm{p}\right)=1\right.$ and the value of $I$ is 0 in all other cases.

(ii) $\mathrm{KT}^{\prime} \mathbf{5}^{\prime}$ is a subsystem of $\mathbf{S 4 . 4}$, since every $\mathbf{S 4 . 4}$ Kripke model is a reflexive transitive semi-euclidean Kripke model. The following KT45' countermodel against the characteristic S4.4 axiom $\varphi \supset\left(\neg K^{\prime} \neg K^{\prime} \varphi \supset K^{\prime} \varphi\right)$ shows that the inclusion is proper: $M^{\prime}=\left\langle w_{0}, W, R^{\prime}, I\right\rangle$, with $\left.R^{\prime}=W \times W \backslash\left(w_{2}, w_{0}\right),\left(w_{2}, w_{1}\right)\right\}$, and $W$ and $I$ as in $M$ of (i).

PROPOSITION 6.7 ROK has ten distinct modalities, viz $\mathrm{K}^{\prime}, \cdot, \mathrm{K}^{\prime} \neg, \mathrm{K}^{\prime} \neg \mathrm{K}^{\prime}, \mathrm{K}^{\prime} \neg \mathrm{K}^{\prime} \neg$, and their negations, with implications among them as pictured below.

Proof. That there are no more modalities follows from $K^{\prime} \varphi \equiv K^{\prime} K^{\prime} \varphi$ and $\neg K^{\prime} \neg K^{\prime} \varphi \supset$ $K^{\prime} \neg K^{\prime} \neg \varphi$. Most of the implications in figure 3 follow from $T(=\square \varphi \supset \varphi) . \neg K^{\prime} \neg K^{\prime} \varphi \supset$ $\mathrm{K}^{\prime} \neg \mathrm{K}^{\prime} \neg \varphi$ follows from $\mathrm{T}$ and $\left(5^{\prime}\right)$. Finally, one can easily construct models showing that the implications pictured in figure 3 are proper.
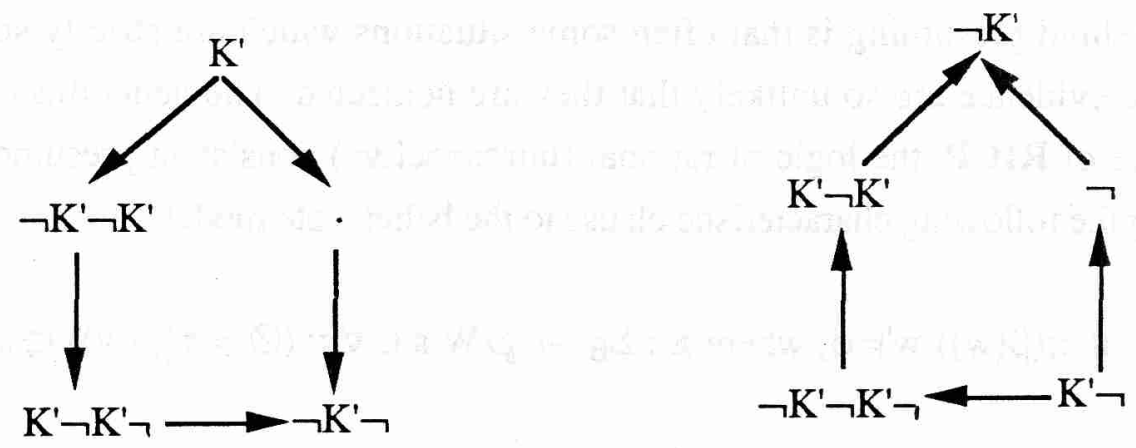

FIGURE 3. Modalities of ROK.

LEMMA 6.8

(i) $\quad \vDash O K \& R I B \neg K^{\prime} \neg K^{\prime} \varphi \equiv B \varphi$

(ii) $\quad \vDash \mathbf{R I B} \neg \mathrm{K}^{*} \neg \mathrm{K}^{*} \varphi \equiv \mathrm{B} \varphi$

Proof. (i) In OK\&RIB we have $\neg \mathrm{B} \varphi \supset \mathrm{K}^{\prime} \neg \mathrm{B} \varphi$ (by 5.8(vi) and negative introspection for $\mathrm{B}$ ) and $K^{\prime} \neg B \varphi \supset K^{\prime} \neg K^{\prime} \varphi$. Hence $\neg B \varphi \supset K^{\prime} \neg K^{\prime} \varphi$. On the other hand, we have $B \varphi \supset K^{\prime} \varphi$ (by (B $\supset$ BK) and positive introspection for B) and $\mathrm{K}^{\prime} \neg \mathrm{K}^{\prime} \varphi \supset \neg \mathrm{BK}^{\prime} \varphi$ (by applying $\mathrm{K}^{\prime} \neg \psi \supset$ $\mathrm{B} \neg \psi$ and $\mathrm{B} \neg \psi \supset \neg \mathrm{B} \psi$ to $\psi=\mathrm{K}^{\prime} \varphi$ ). Hence $\mathrm{K}^{\prime} \neg \mathrm{K}^{\prime} \varphi \supset \neg \mathrm{B} \varphi$. (ii) Similar. 
Wolfgang Lenzen suggested that for every reasonable notion of knowledge $K$ and belief $B$ the equivalence $B \varphi \equiv \neg K \neg K \varphi$ should hold. Additionally assuming $K \varphi \supset \varphi$ and $K \varphi \supset K K \varphi$ he arrived at his already mentioned conclusion that the logic of knowledge is at least as strong as S4.2.

\section{Weak notions of belief}

The notion of rational introspective belief of section 4 is a very strong notion of belief. In this section we will consider some weaker versions of belief which can still be considered rational and introspective. We take serious the remark in section 4 that in general a rational belief state $S$ should not be equated with IIS and we consider some modalities which refer to the (relative) likelihood of subclasses of $\|S\|$.

Consider $\mathrm{P}_{\alpha}$ with the following intuitive meaning

$\mathrm{P}_{\alpha} \varphi$ : agent $\alpha$ presumes $\varphi$, i.e. $\varphi$ is true in every possible world which $\alpha$ considers to be not too unlikely.

The rationale behind presuming is that often some situations which are strictly speaking not excluded by the evidence are so unlikely that they are neglected. The generalized functional model semantics of RICP, the logic of rational (introspective) consistent presumed belief, is given by adding the following characteristic clause to the belief state models:

$w \models P \varphi \Leftrightarrow \forall w^{\prime} \in \pi(\beta(w)) w^{\prime} \vDash \varphi$, where $\pi: \Sigma_{B} \rightarrow \wp W$ s.t. $\forall w(\varnothing \neq \pi(\beta(w)) \subseteq\|\beta(w)\|)$

Here $\pi(\beta(w))$ denotes the set of worlds which are not neglected. The requirement $\pi(\beta(w)) \neq \varnothing$ ensures that the presumed belief is consistent. If one drops this requirement, one obtains the semantics of RIP, the logic of (introspective) presumed belief. One easily obtains a functional model semantics for $\mathbf{R I}(\mathbf{C}) \mathbf{P}$, which is equivalent to its generalized functional model semantics:

\section{DEFINITION 7.1}

(i) A RIP-frame is a functional frame $\mathrm{F}=<\mathrm{W}, \beta, \pi>$ for $\mathcal{L}_{\mathrm{BP}}$ such that

1. $\forall w \pi(w) \subseteq \beta(w)$

2. $\forall w, w^{\prime} \in W\left(\beta(w)=\beta\left(w^{\prime}\right) \Rightarrow \pi(w)=\pi\left(w^{\prime}\right)\right)$ 
(ii) A RIP-model is a functional model based on a RIP-frame.

(iii) A RICP-model/frame is an $\boldsymbol{R I P}$-model/frame with the additional assumption that $\pi(\mathrm{w}) \neq$ $\varnothing$.

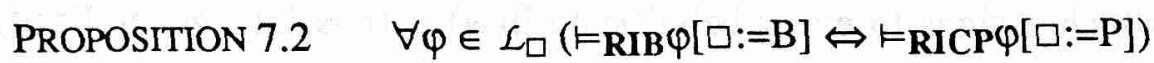

Proof. Consider a RIB-frame $\mathrm{F}=\left\langle\mathrm{W}, \beta>\right.$. Then $\mathrm{F}^{\prime}=\langle\mathrm{W}, \beta, \beta>$ is a RICP-frame such that the valid pure $\mathrm{P}$-formulas in $\mathrm{F}^{\prime}$ correspond with the valid pure $\mathrm{B}$-formulas in $\mathrm{F}$. On the other hand, if $F=\left\langle W, \beta, \pi>\right.$ is RICP-frame, then $F^{\prime}=\langle W, \pi>$ is a RIB-frame such that the valid pure Bformulas in $\mathrm{F}$ correspond with the valid pure $\mathrm{P}$-formulas in $\mathrm{F}$.

Of course the above proposition does not imply that there is no difference between B and P. However, this difference will only show in contexts where these operators are combined with each other or other operators. For example, it is easy to see that (with respect to the obvious semantics) $\mathrm{B}(\mathrm{P} \varphi \supset \varphi)$ and $\mathrm{P} \varphi \supset \mathrm{PK} \varphi$ are invalid, whereas $\mathrm{B}(\mathrm{B} \varphi \supset \varphi)$ and $\mathrm{B} \varphi \supset \mathrm{BK} \varphi$ are valid.

Next consider the notion $\mathrm{L}_{\alpha}$ with the following intuitive meaning:

$\mathrm{L}_{\alpha} \varphi$ : agent $\alpha$ finds it likely that $\varphi$, i.e. $\varphi$ is true in every world of some subclass of $\|\beta(w)\|$ which is considered to be likely by $\alpha$.

This notion of likely belief should not be confused with the notion of likelihood (also denoted by $\mathbf{L}$ ) which is formalized in the system $\mathbf{L L}$ of Halpern \& Rabin (1987). LL tries to capture the notion of being likely in a nonnumerical way. The characteristic clause of the semantics of RIL, the logic of rational introspective likely belief, is the following:

$w \models L \varphi \Leftrightarrow \exists A \in \lambda(\beta(w)) \forall w^{\prime} \in A w^{\prime} \models \varphi$, where $\lambda: \Sigma_{\mathrm{B}} \rightarrow \wp^{*}\left(\wp^{*}(\|\beta(w)\|)\right)$.

$(\wp *(\mathrm{X})$ denotes $\wp(\mathrm{X}) \backslash\{\varnothing\}$.

We have that $\vDash_{\mathbf{R I L}} \varphi \supset \psi$ implies that $\vDash_{\mathbf{R I L}} L \varphi \supset \mathbf{L} \psi$, hence $\mathbf{R I L}$ is a classical modal logic (in the sense of Segerberg (1971)). However, RIL is not normal and not even regular, since neither $\mathrm{L}(\varphi \supset \psi) \supset(\mathrm{L} \varphi \supset \mathrm{L} \psi)$ nor $(\mathrm{L} \varphi \wedge \mathrm{L} \psi) \supset \mathrm{L}(\varphi \wedge \psi)$ is valid. Hence there is no standard Kripke model semantics or functional model semantics for RIL. (There does, of course, exist a neighbourhood model semantics for RIL.) From the assumption that only non-empty sets can be considered likely it follows that $L \neg \varphi \supset \neg L \varphi$ is valid. As a consequence of the introspection condition for rational belief states we further have $\mathrm{L}(\neg) \mathrm{B} \varphi \equiv(\neg) \mathrm{B} \varphi, \mathrm{L}(\neg) \mathrm{L} \varphi \equiv(\neg) \mathrm{L} \varphi$, and 
$\mathrm{B}(\neg) \mathrm{L} \varphi \equiv(\neg) \mathrm{L} \varphi$. (Hence different powers of L do not denote different levels of likelihood, as is the case in $\mathbf{L L}$.)

A plausible reading of $L_{\alpha} \varphi$ is " $\alpha$ 's subjective probability of $\varphi$ is at least $x$ ", for some particular $x \in(0,1]$. An alternative semantics for this notion can be obtained by using the projection function $\Pi$ which assigns to every belief state $\beta(w)$ a (possibly generalized) probability measure $P_{w}$ defined on (a $\sigma$-algebra/field $\mathcal{F}_{w}$ of subsets of) $\|\beta(w)\|$. In that case one may define for some $x \in(0,1]$ :

$w \models L \varphi \Leftrightarrow P_{w}\left(\left\{w^{\prime} \in\|\beta(w)\| \mid w^{\prime} \vDash \varphi\right\}\right) \geq x$ or $\mathrm{w} \models \mathrm{L} \varphi \Leftrightarrow \exists \mathrm{A} \in \mathcal{F}_{\mathrm{W}} \forall \mathrm{w}^{\prime} \in \mathrm{A}\left(\mathrm{w}^{\prime} \vDash \varphi\right.$ and $\left.\mathrm{P}_{\mathrm{w}}(\mathrm{A}) \geq \mathrm{x}\right)$.

This semantics can easily be extended to interpret a richer formalism of rational subjective probability (RSP):

$w \vDash P \geq x \varphi P_{w}\left(\left\{w^{\prime} \in\|\beta(w)\| \mid w^{\prime}=\varphi\right\}\right) \geq x$.

$\left(\mathrm{P} \leq \mathrm{x} \varphi, \mathrm{P}^{\mathrm{x}} \varphi\right.$ and $\mathrm{P}<\mathrm{x} \varphi$ are defined analogously.)

In general, the relation between knowledge/belief and probability is a far from trivial matter. (Consider for example the problem mentioned in Fagin \& Halpern (1988) of assigning the appropriate probability spaces.) Next to the notion of rational subjective belief, one has a notion of objective probability, and a 'subjectivized' notion of objective probability (the objective probability given the objective information available to a particular agent), and then some more. All the more reason to look for instruments which allow one to faithfully formalize the multitude of (different combinations of) epistemic notions. The generalized functional models might be considered to be such instruments, although they probably have to be sharpened in order to really cut through.

\section{Conclusion}

We introduced generalized functional models which generalize Kripke models by explicitly representing epistemic states. Those generalized functional models were used to identify the notions of objective knowledge and rational (introspective) belief and to determine the logical relation between the two notions. Although objective knowledge and rational belief seem to correspond to the predominant notions of (S5-)knowledge and (KD45-)belief, the system 
OK\&RIB combining the two notions turned out to be quite different from the well-known system (KL) of Kraus and Lehmann. We argued against KL by showing that negative introspection for knowledge is incompatible with the principle that knowledge implies belief (assuming beliefs to be consistent). Combining an S5-notion of knowledge with an KD45notion of belief thus means dropping the knowledge-implies-belief-principle. Further, we briefly considered some notions of knowledge which imply belief and some weak notions of rational belief, partially as an illustration for the need of something like generalized functional models to keep track of the multitude of epistemic notions.

\section{Acknowledgments}

Albert Visser substantially influenced my ideas on the subject of this paper and members of the Amsterdam Working Group on Nonmonotonic Reasoning kindly commented on an early unpolished presentation of these ideas.

The investigations were supported by the Foundation for Philosophical Research (SWON), which is subsidized by the Netherlands Organization for Scientific Research (NWO).

\section{References}

R. FAGIN \& J. HALPERN, 'Belief, Awareness, and Limited Reasoning', Artificial Intelligence 34 (1988) 39-76.

R. FAGIN \& J. HALPERN, 'Reasoning About Knowledge and Probability: Preliminary Report,' in M. Vardi (ed.), Theoretical Aspects of Reasoning About Knowledge: Proceedings of the Second Conference, Morgan Kaufmann, Los Altos Ca. (1988) 277-293

J. HALPERN (ed.), Theoretical Aspects of Reasoning About Knowledge: Proceedings of the 1986 Conference, Morgan Kaufmann, Los Altos Ca. (1986)

J. HALPERN \& M. RABIN, 'A Logic to Reason about Likelihood', Artificial Intelligence 32 (1987) 379-405.

J. HINTIKKA, Knowledge and Belief: An Introduction to the Logic of the Two Notions, Cornell U.P., Ithaca (1962).

W. VAN DER HOEK, 'Systems for Knowledge and Beliefs', rapport IR-185 Free University Amsterdam (1989). To appear in Proceedings JELIA 1990.

S. Kraus \& D. LehMANN, 'Knowledge, belief and time', Proc. 13th ICALP, C. Krott (ed.), Springer, Berlin (1986) 186-195.

W. LeNZEN, Recent work in epistemic logic, Acta Philosophica Fennica 30, North-Holland, Amsterdam (1978).

K. Segerberg, An Essay in Classical Modal Logic, vol. 1-3, Philosophical Studies 13, University of Uppsala (1971) 


\section{Logic Group Preprint Series \\ Department of Philosophy \\ University of Utrecht \\ Heidelberglaan 2 \\ 3584 CS Utrecht \\ The Netherlands}

1 C.P.J. Koymans, J.L.M. Vrancken, Extending Process Algebra with the empty process, September 1985

2 J.A. Bergstra, A process creation mechanism in Process Algebra, September 1985

3 J.A. Bergstra, Put and get, primitives for synchronous unreliable message passing, October 1985

4 A. Visser, Evaluation, provably deductive equivalence in Heyting's arithmetic of substitution instances of propositional formulas, November 1985

5 G.R. Renardel de Lavalette, Interpolation in a fragment of intuitionistic propositional logic, January 1986

6 C.P.J. Koymans, J.C. Mulder, A modular approach to protocol verification using Process Algebra, April 1986

7 D. van Dalen, F.J. de Vries, Intuitionistic free abelian groups, April 1986

8 F. Voorbraak, A simplification of the completeness proofs for Guaspari and Solovay's R, May 1986

9 H.B.M. Jonkers, C.P.J. Koymans \& G.R. Renardel de Lavalette, A semantic framework for the COLD-family of languages, May 1986

10 G.R. Renardel de Lavalette, Strictheidsanalyse, May 1986

11 A. Visser, Kunnen wij elke machine verslaan? Beschouwingen rondom Lucas'argument, July 1986

12 E.C.W. Krabbe, Naess's dichotomy of tenability and relevance, June 1986

$13 \mathrm{H}$. van Ditmarsch, Abstractie in wiskunde, expertsystemen en argumentatie, Augustus 1986

14 A. Visser, Peano's Smart Children, a provability logical study of systems with built-in consistency, October 1986

15 G.R. Renardel de Lavalette, Interpolation in natural fragments of intuitionistic propositional logic, October 1986

16 J.A. Bergstra, Module Algebra for relational specifications, November 1986

17 F.P.J.M. Voorbraak, Tensed Intuitionistic Logic, January 1987

18 J.A. Bergstra, J. Tiuryn, Process Algebra semantics for queues, January 1987

19 F.J. de Vries, A functional program for the fast Fourier transform, March 1987

20 A. Visser, A course in bimodal provability logic, May 1987

21 F.P.J.M. Voorbraak, The logic of actual obligation, an alternative approach to deontic logic, May 1987

22 E.C.W. Krabbe, Creative reasoning in formal discussion, June 1987

23 F.J. de Vries, A functional program for Gaussian elimination, September 1987

24 G.R. Renardel de Lavalette, Interpolation in fragments of intuitionistic propositional logic, October 1987 (revised version of no. 15)

25 F.J. de Vries, Applications of constructive logic to sheaf constructions in toposes, October 1987

26 F.P.J.M. Voorbraak, Redeneren met onzekerheid in expertsystemen, November 1987

27 P.H. Rodenburg, D.J. Hoekzema, Specification of the fast Fourier transform algorithm as a term rewriting system, December 1987 
28 D. van Dalen, The war of the frogs and the mice, or the crisis of the Mathematische Annalen, December 1987

29 A. Visser, Preliminary Notes on Interpretability Logic, January 1988

30 D.J. Hoekzema, P.H. Rodenburg, Gauß elimination as a term rewriting system, January 1988

31 C. Smoryński, Hilbert's Programme, January 1988

32 G.R. Renardel de Lavalette, Modularisation, Parameterisation, Interpolation, January 1988

33 G.R. Renardel de Lavalette, Strictness analysis for POLYREC, a language with polymorphic and recursive types, March 1988

34 A. Visser, A Descending Hierarchy of Reflection Principles, April 1988

35 F.P.J.M. Voorbraak, A computationally efficient approximation of Dempster-Shafer theory, April 1988

36 C. Smoryński, Arithmetic Analogues of McAloon's Unique Rosser Sentences, April 1988

37 P.H. Rodenburg, F.J. van der Linden, Manufacturing a cartesian closed category with exactly two objects, May 1988

38 P.H. Rodenburg, J.L.M.Vrancken, Parallel object-oriented term rewriting : The Booleans, July 1988

39 D. de Jongh, L. Hendriks, G.R. Renardel de Lavalette, Computations in fragments of intuitionistic propositional logic, July 1988

40 A. Visser, Interpretability Logic, September 1988

41 M. Doorman, The existence property in the presence of function symbols, October 1988

42 F. Voorbraak, On the justification of Dempster's rule of combination, December 1988

43 A. Visser, An inside view of EXP, or: The closed fragment of the provability logic of $I \Delta_{0}+\Omega_{1}$, February 1989

44 D.H.J. de Jongh \& A. Visser, Explicit Fixed Points in Interpretability Logic, March 1989

45 S. van Denneheuvel \& G.R. Renardel de Lavalette, Normalisation of database expressions involving calculations, March 1989

46 M.F.J. Drossaers, A Perceptron Network Theorem Prover for the Propositional Calculus, July 1989

47 A. Visser, The Formalization of Interpretability, August 1989

48 J.L.M. Vrancken, Parallel Object Oriented Term Rewriting : a first implementation in Pool2, September 1989

49 G.R. Renardel de Lavalette, Choice in applicative theories, September 1989

50 C.P.J. Koymans \& G.R. Renardel de Lavalette, Inductive definitions in COLD-K, September 1989

51 F. Voorbraak, Conditionals, probability, and belief revision (preliminary version), October 1989

52 A. Visser, On the $\Sigma_{1}^{0}$-Conservativity of $\Sigma_{1}^{0}$-Completeness, October 1989

53 G.R. Renardel de Lavalette, Counterexamples in applicative theories with choice, January 1990

54 D. van Dalen, L.E.J. Brouwer. Wiskundige en Mysticus, June 1990

55 F. Voorbraak, The logic of objective knowledge and rational belief, september 1990 